\title{
Development of a Superconducting Magnetic Bearing Capable of Supporting Large Loads in a Flywheel Energy Storage System for Railway Application
}

\author{
$\begin{array}{lll}\text { Yoshiki MIYAZAKI Katsutoshi MIZUNO Mafumi OGATA } & \text { Mafurat }\end{array}$ \\ Cryogenic Systems Laboratory, Maglev Systems Technology Division \\ Tomohisa YAMASHITA \\ Ken NAGASHIMA \\ Maglev Systems Technology Division
}

\begin{abstract}
Application of the flywheel energy storage system (FESS) using high temperature superconducting magnetic bearings (SMB) has been demonstrated at the Komekurayama photovoltaic power plant located in Yamanashi Prefecture. In order for the FESS to be applied to railways as a system able to prevent cancellation of regenerative braking its storage capacity must be increased. Therefore, levitation force tests up to $158 \mathrm{kN}$ and tests to determine the creep characteristics of the levitation force were carried out to verify the margin in SMB levitation force. Furthermore, to evaluate long-term reliability and durability of the levitation and rotation characteristics of the SMB against repeated change in rotation speed, new SMB test apparatus capable of simultaneously testing both the levitating and rotating states of the SMB is being developed.
\end{abstract}

Keywords: flywheel, energy storage system, superconducting magnetic bearing, rail application, large load

\section{Introduction}

Flywheels are a promising storage system for high frequency charge/discharge cycles which can prevent voltage drops in railway overhead line, or collect regenerative energy from braking trains. The Railway Technical Research Institute has already proposed a superconducting magnetic bearing with high-temperature superconducting coils and bulks which can support a large load stably without contact [1]. The contactless SMB improves maintainability, which has been a problem in conventional mechanical bearings. Results from tests have been collected on a prototype FESS using a SMB installed at the Komekurayama photovoltaic power plant in Yamanashi prefecture [2][3][4]. The SMB used in the FESS at the Komekurayama plant has remained in a levitating state for 9500 hours without any problems, confirming the reliability and stability of the SMB (Fig. 1). The levitation force of the Komekurayama SMB is $39 \mathrm{kN}$. However, for application on the railways, a larger storage capacity is required. Consequently, the SMB had to be improved to support a larger load. Subsequent tests confirmed that the improved SMB was able to support a larger load up to $158 \mathrm{kN}$, commensurate with increase required for the storage capacity of the flywheel to be applicable to railway systems [4]. This report describes the levitation force verification test, and the creep characteristic test conducted up to $158 \mathrm{kN}$, which confirmed the levitation force characteristics of the SMB. The test equipment used to evaluate the reliability and durability of the SMB and cryo-materials employed are also described.

\section{Flywheel energy storage system}

\subsection{Principle of FESS}

Flywheel energy storage systems can store electricity in the form of kinetic energy by rotating a flywheel. By converting kinetic energy to electric energy it is able to reconvert this energy into electricity again on demand. FESSs do not deteriorate in the way of chemical cells due to repeated charging and discharging. Consequently, the FESS is suitable for long-life storage systems which have high frequency charging/discharging cycles. For example, for an expected serviceable life of 30 years, a FESS must withstand several million or more charge/discharge cycles, in order to effectively utilize the regenerative energy produced by electric railways or to moderate electrical power fluctuations from renewable energy sources, such as photovoltaic and wind power plants. As such, the FESS is considered to be an option for such applications. In addition, given that FESSs do not deteriorate in the same way as chemical cells through repeated charge/discharge cycles, there is no need for back-up installations or to change cells. Furthermore, output power and stored capacity in the chemical cells or capacitors have a fixed relationship so they cannot be set independently. With the FESS however, output power is determined by the motor-generator and stored capacity is determined by the mass, radius and rotational speed of the flywheel, which can be set independently. Furthermore, by monitoring the rotational speed it is possible to check the remaining stored capacity at any

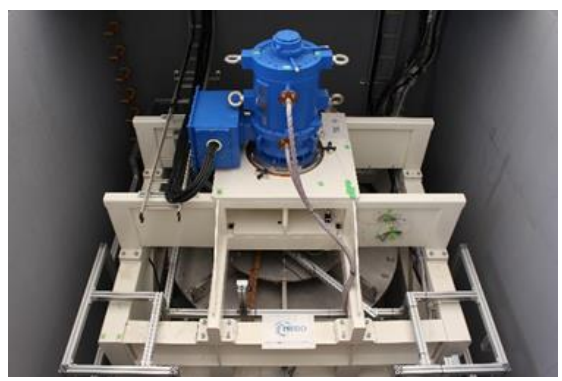

Fig. 1 Overview of Komekurayama flywheel prototype 
moment, and the totality of the stored energy can be reconverted again into electricity by the time the flywheel stops rotating.

\subsection{Configuration of the FESS with SMB}

Figure 2 shows the components in the Komekurayama FESS prototype with a SMB. The FESS consists of a SMB, a flywheel, an active magnetic bearing (AMB), a magnetic fluid seal and a generator/motor. The flywheel, which stores energy by rotation is made of CrMoNi steel and has a diameter of $1.44 \mathrm{~m}$, a thickness of $0.29 \mathrm{~m}$, and a mass of $4000 \mathrm{~kg}$. The thrust load of the flywheel is supported by the SMB consisting of the HTS coils and bulks. The generator/motor converts kinetic energy into electrical energy and vice versa. The generator/motor is located outside the vacuum vessel, and the vacuum vessel has a magnetic fluid seal capable of maintaining a vacuum even while rotating at high speed. The imbalance of the flywheel rotor was reduced to $40 \mathrm{~g}$ below the target. In the rotation speed improvement test, it reached the maximum rotation speed of $3000 \mathrm{~min}^{-1}$ without any field balance adjustments [5].

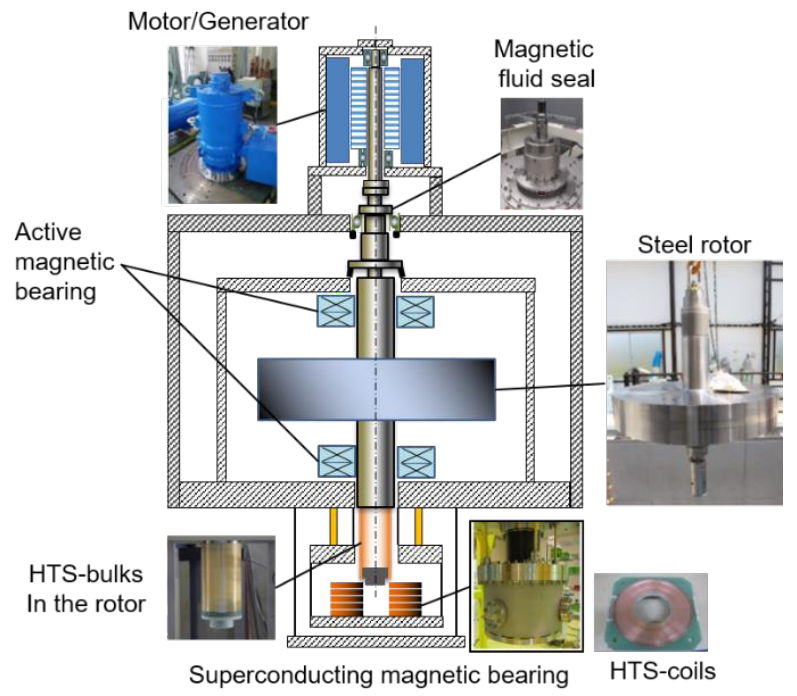

Fig. 2 Flywheel energy storage system with SMB

\section{SMB capable of supporting a large load}

\subsection{SMB configuration and levitation principle}

In general, mechanical bearings in flywheels support a large load periodic bearing maintenance is necessary. Therefore, conventional flywheel performance is inferior to chemical cells or capacitors because of this maintenance, which is the reason why flywheels have not become commercially mainstream. RTRI managed to overcome this problem by developing a contactless SMB to support the flywheel with strong magnetic repulsive forces that avoid mechanical losses. Figure 3 shows the basic components of a SMB. The SMB has HTS bulks in the rotor and HTS coils as a stator. First, HTS bulks are cooled without excitation (zero-field cooling) and brought into a superconducting state. Then, as the HTS coils are excited, a large magnetic field is generated around the HTS bulks. The shielding current on the surface of the HTS bulks excludes the magnetic flux trying to cancel the magnetic field. As a result, a large magnetic repulsive force is generated between the HTS bulks and coils. This repulsive force is the levitation force, and is proportional to the square of the current of the HTS coils: consequently, since theoretically no force acts in a radial direction, and because of the rotational symmetry of the HTS bulks and coils are rotational symmetry, therefore, the SMB achieves perfect levitation without any frictional losses. The HTS coils are cooled directly by the thermally connected refrigerator, however, the HTS bulks installed in the rotational rotor cannot be cooled by the refrigerator directly, therefore they are cooled indirectly by helium gas filling the inner vessel of the SMB. The pressure of the helium gas in the inner vessel is set in the pressure range (around $10 \mathrm{~Pa}$ ) to achieve low gas friction and large heat transfer [6]. This approach therefore provides a simple means to cool the SMB without using a coolant such as liquid nitrogen.

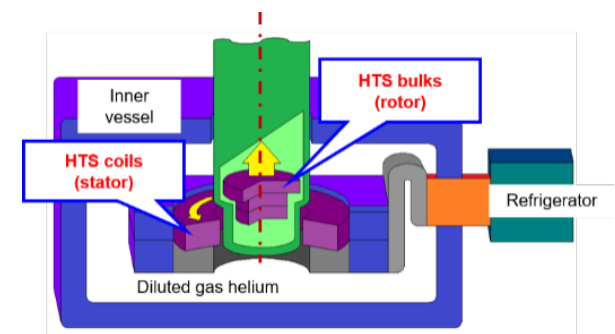

Fig. 3 HTS coils and bulks constituting the SMB

\subsection{HTS coil}

The HTS coil specifications are given in Table 1. The HTS coils forming the stator comprise 5 double pancakelike coils with an inner diameter of $120 \mathrm{~mm}$, and outer diameter of $260 \mathrm{~mm}$, the coils are wound with REBCO tape wire $6 \mathrm{~mm}$ in width and $0.1 \mathrm{~mm}$ thick. The design of the HTS coils was based on the latest superconducting wire properties, taking into account the HTS load factor, and fixed with a thermoplastic resin, based on actually developed large-scale high-temperature superconducting coils used on Maglev railways, to improve the heat conductivity of the superconducting coil (Fig.4) [7][8][9][10].

Table 1 Specifications of HTS coils and bulks

\begin{tabular}{llr}
\hline Item & \multicolumn{2}{c}{ Specifications } \\
\hline Wire & Type & REBCO by SuperPower Inc. \\
& Wilikh [mm] & 6 \\
& Thickness [mm] & 0.1 \\
\hline Coil & Inner diameter [mm] & 120 \\
& Outer diameter [mm] & 6 \\
& Thickness/double pancake [mm] & 17.6 \\
& Number of double pancakes & 5 \\
& Inductance [H] & 4 \\
\hline Bulk & Type & REBCO by Nippon Steel Corp. \\
& Diameter of large bulk [mm] & 140 \\
& Diameter of small bulk [mm] & 90 \\
& Thickness [mm] & 20 \\
\hline
\end{tabular}




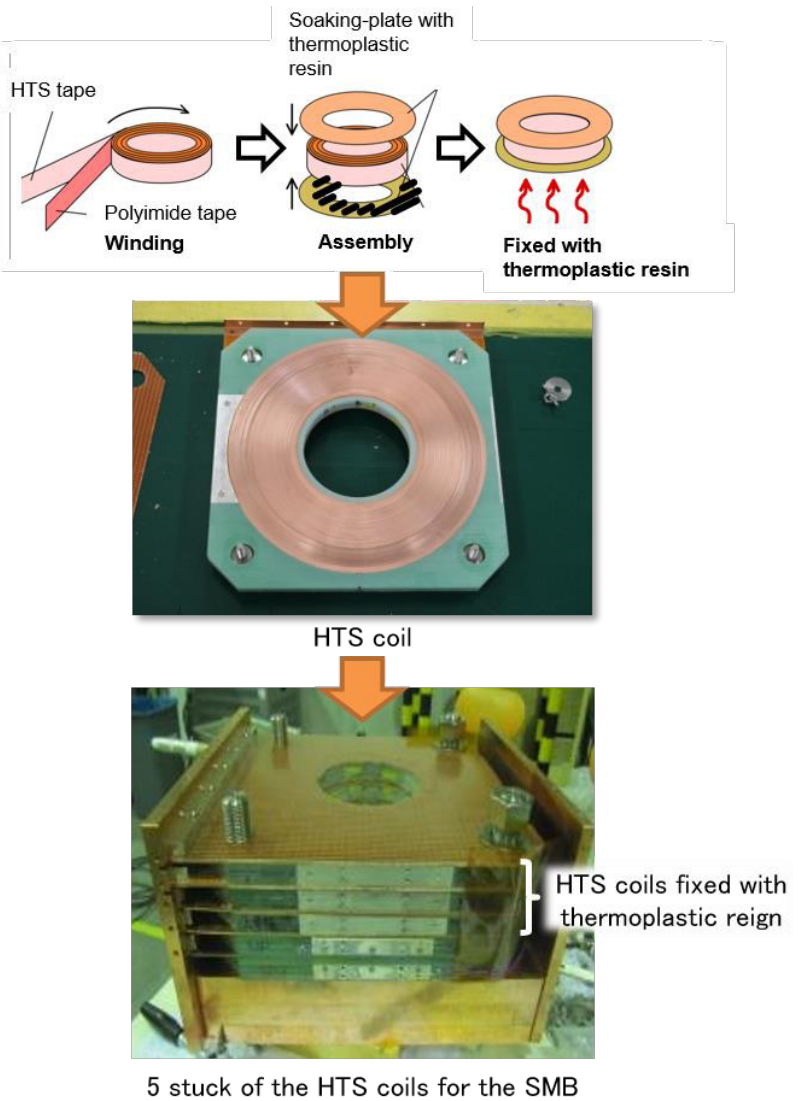

Fig. 4 New HTS coils fixed with thermoplastic reign

\subsection{HTS bulk}

One HTS bulk with a diameter of $140 \mathrm{~mm}$ and two HTS bulks with a diameter of $90 \mathrm{~mm}$ were stacked in the rotor (Fig. 5). The HTS bulks were manufactured by NIPPON STEEL Corporation using the advanced "Quench and Melt Growth" method and were made in the shape of a large disk [11][12]. The HTS bulk with a diameter of 140 mm mainly supports the thrust load, while the two HTS bulks with a diameter of $90 \mathrm{~mm}$ function as a guide in the radial direction.

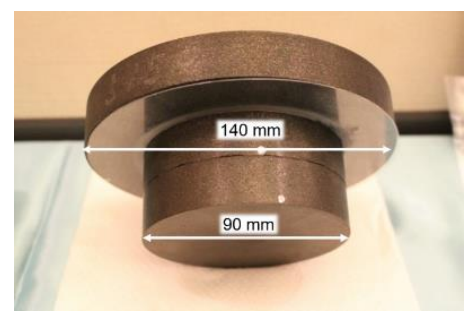

Fig. 5 Flywheel rotor HTS bulks

\section{SMB levitation tests}

\subsection{Confirmation of levitation force margin}

A levitation force test up to $158 \mathrm{kN}$ was conducted to confirm the margin in the SMB performance. Figure 6 shows the experimental setup for the levitation test of the SMB [4][13]. The test equipment had the same components
(HTS bulks in the rotor, HTS coils, thermally insulated supports supporting the magnetic repulsive force between the HTS coils and bulks, inner and outer vessels) as the Komekurayama prototype. The levitation force was measured using a load cell outside the vacuum vessel because the test equipment did not have a flywheel rotor. The test conditions were the same as the $147 \mathrm{kN}$ levitation test: after cooling the HTS coils and bulks in the helium gas atmosphere, the HTS coils were excited [4]. Figure 7 shows the result of the levitation test. The levitation force was in equilibrium with the atmospheric pressure load $(0.93 \mathrm{kN})$ when the applied current exceeded $20 \mathrm{~A}$, and in the region above $25 \mathrm{~A}$, the levitation force increased almost by the square of the applied current, and the levitation force of the target value of $158 \mathrm{kN}$ was obtained when the applied current was $156 \mathrm{~A}$. The reason for the hysteresis between the magnetization and demagnetization is considered to be the influence of the magnetic flux penetration into the HTS bulks. From these results, it can be demonstrated that a high load SMB using a new HTS coil fixed with a thermoplastic resin is established.

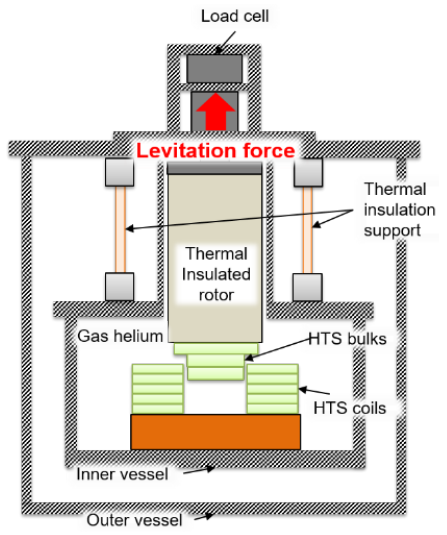

\section{Fig. 6 Experimental setup for large load levitation test of} SMB

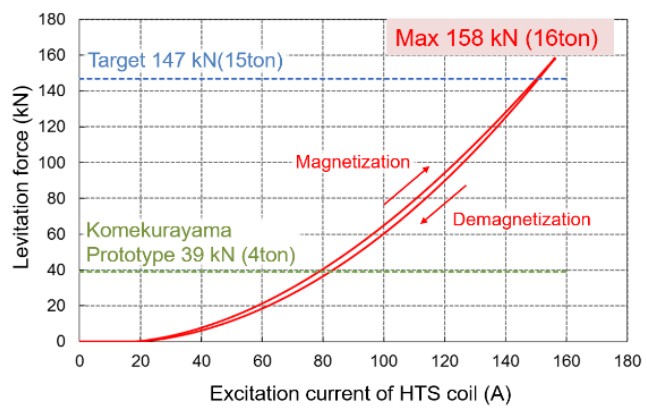

Fig. 7 Result of SMB large load levitation test

\subsection{Continual 8-hour levitation test}

It is known that levitation force decreases with time when an external magnetic field is applied to superconducting bulks. This phenomenon is similar to the one where magnetization of permanent magnets decreases over time, which is known as "magnetic after effect" or "flux creep" [14][15][16]. A continual levitation test over $148 \mathrm{kN}$ at 151 A for 8 hours was conducted twice to comprehend the initial properties of the flux creep (Fig. 8). Figure 8 shows that the 


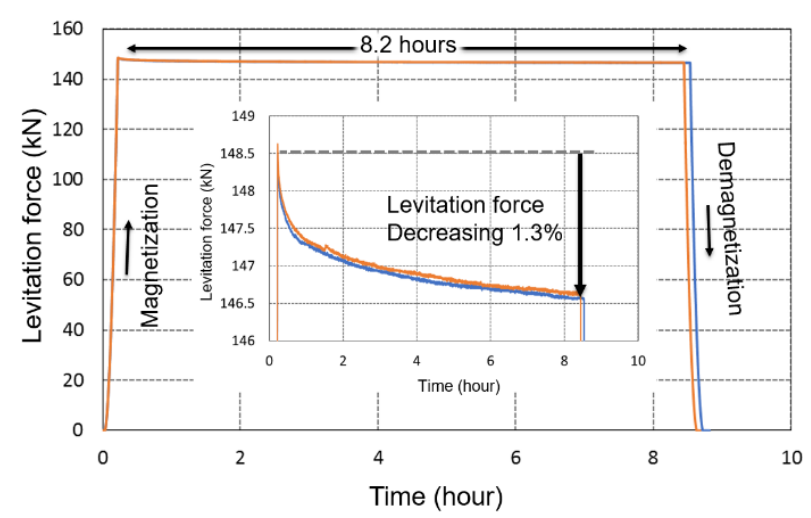

Fig. 8 Result of continual levitation test for 8 hours

flux creep showed the same tendencies in both tests, and the test results suggest good repeatability without the influence of the levitation force hysteresis with excitation. The enlarged graph in the figure shows that the attenuation of the levitation force after 8 hours was just over 1\%, and that the decrease in levitation force decreased with time. It is thought that the flux leak into superconducting bulks causes the levitation force to decrease. Considering the relationship between the flux leak and the levitation force, and the decreasing characteristics of shielding current around the superconducting bulks, the levitation force decreasing is calculated as $3 \%$ a month or around $4 \%$ a year. In the Komekurayama flywheel prototype, the levitation height of the flywheel is kept constant by controlling the current applied to the superconducting coils in consideration of the occurrence of such a flux creep phenomenon. Based on the experience of the Komekurayama flywheel prototype, it was confirmed that the levitation height of the flywheel could be kept constant by controlling the current applied to the superconducting coils, if the flux creep characteristics are at this level even when the load to be supported is increased.

\section{Soundness of HTS coils and SMB supports}

\subsection{Excitation characteristics of HTS coils after levi- tation tests}

In order to confirm the soundness of the HTS coils, the critical current of the HTS coils and the $n$ value, which shows the characteristics of superconductor, were measured. To calculate the $\mathrm{n}$ values, a fitting curve was made from the results of the I-V properties of each doublepancake coil when the HTS coils were excited. The exponent part of the curve was defined as the $n$ value, and the current value at the generated voltage of $1 \mathrm{mV}$ was defined as the critical current value. Each double-pancake coil was excited in the liquid nitrogen after precooling those by boiling gas nitrogen in the cryo-vessel (Fig. 9). Figure 10 shows the comparison of the critical currents and $n$ values before and after the levitation test. 'Before the test' means the factory-gate properties and 'after the test' refers to properties found after the series of $158 \mathrm{kN}$ levitation tests. The characteristics after the test were the same as before the test, and it was confirmed that there was no change in characteristics before and after the $158 \mathrm{kN}$ levitation tests.

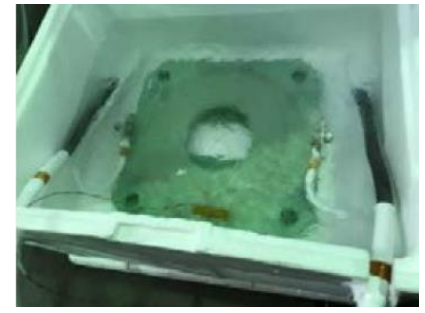

Fig. 9 HTS coil current test in liquid nitrogen

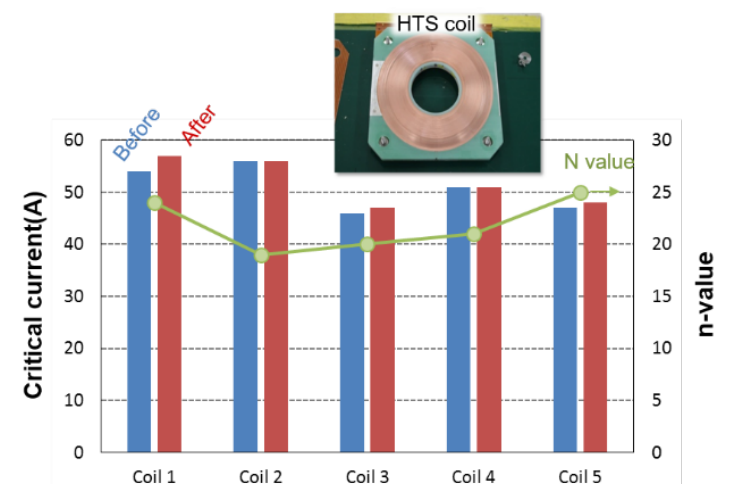

Fig. 10 Comparison of the critical currents and $\mathbf{n}$ values before and after the levitation test

\subsection{Tension test of the SMB supports}

After the $158 \mathrm{kN}$ levitation tests, tension tests were conducted on the four SMB supports, which also demonstrated that their characteristics before and after the tests did not change. Alumina fiber-reinforced plastic which has good thermal insulation and strength was used as the SMB support. Strain gauges were set to the center of the AFRP rods [17]. Figure 11 shows the result of the tension test of the SMB supports. Figure 12 shows the Young's modulus calculated from the result of the stress-strain test. The Young's modulus of each SMB support did not change significantly before and after the tests, and it was confirmed that there was no deterioration due to the large load levitation force, as in the superconducting coil.
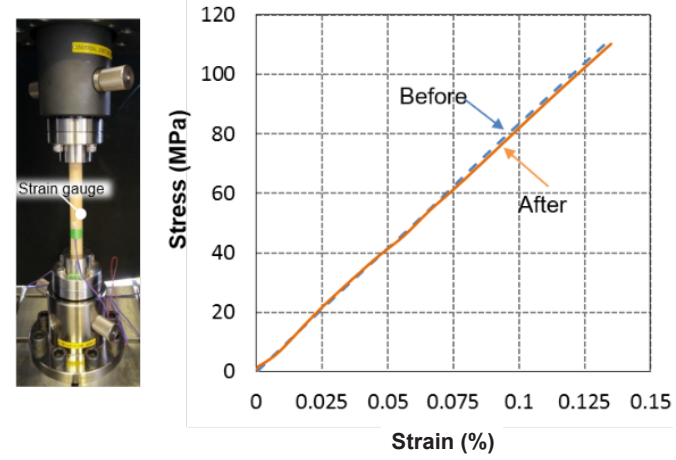

Fig. 11 Results of tension test of SMB support before and after the levitation test 


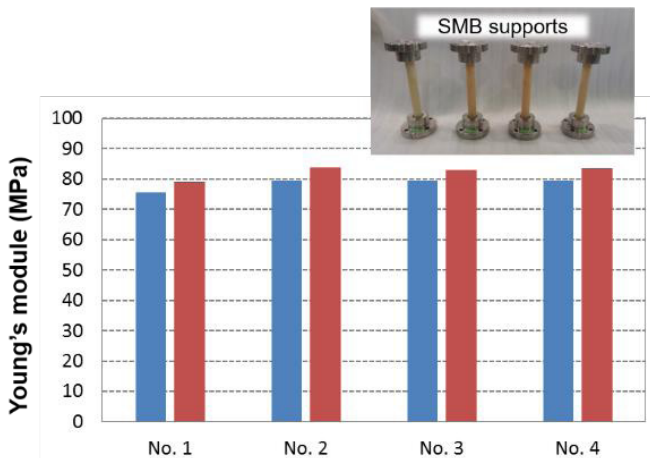

Fig. 12 Comparison of the Young's modulus of SMB supports before and after the levitation test

\section{Design of the SMB reliability and durability test equipment}

It is estimated that the number of charge/discharge cycles required for the energy storage systems to prevent voltage drops or to be able to effectively collect regenerative energy from braking trains, is to be several hundred thousand to several million. As mentioned in chapter 4, the levitation test of the SMB was a static evaluation without rotation of the flywheel, however, the stable levitation and rotation of the SMB needs to be evaluated dynamically to understand its performance with repeated changes in rotational speed. Below is a description of the experimental equipment used for acceleration tests that allow simultaneous levitation and rotation, and can evaluate the long term reliability and durability of the HTS coils and cryo-parts of the SMB similar to the Komekurayama prototype. The SMB reliability and durability evaluation test equipment was required to:

(1) Achieve rotation and levitation simultaneously for dynamic verification;

(2) Perform rapid accelerations and decelerations for SMB component acceleration tests;

(3) Give forced oscillation to the rotor for evaluation of vibration resistance performance.

In the SMB reliability and durability evaluation test equipment, SMBs were arranged in vertically symmetrical position with respect to the rotor, and the magnitude of the force was the same on the rotor so that a reverse force was generated cancelling the SMBs' forces (Fig. 13). In this way, it was possible to create a state where the levitation force necessary for the test was generated even if the mass of the rotor was small.

Currently, evaluation equipment is being developed that can accelerate/decelerate to a maximum speed of 3600 $\min ^{-1}$ with a load of $147 \mathrm{kN}$ and can perform approximately $10 \times 5$ acceleration tests corresponding to the assumed number of charge/discharge cycles. In addition, this evaluation equipment can also conduct a performance evaluation of the touch down bearing, which is a safety device on the flywheel energy storage system.

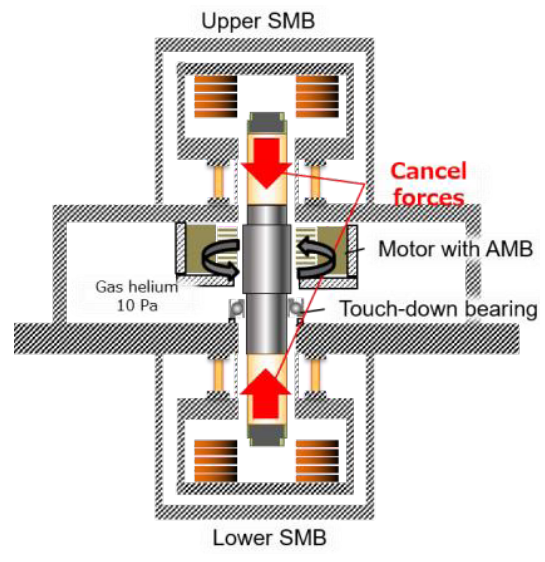

Fig. 13 SMB reliability and durability test equipment

\section{Conclusions}

Levitation tests to a maximum of $158 \mathrm{kN}$ and flux creep tests were conducted to verify the margin of levitation force and characteristics of the SMB used on a flywheel for used on railways. Tests to verify the SMB levitation force margin, confirmed a stable levitation of 158 $\mathrm{kN}, 7 \%$ over the target value. In the flux creep test, the decrease of the SMB levitation force was $1.3 \%$ for 8 hours, and it was estimated to be $4 \%$ over a year. The levitation height of the flywheel can be kept constant by controlling the current applied to the superconducting coils if the flux creep characteristics are at this level. After the large load levitation test of the SMB, the critical current of the HTS coils and $\mathrm{n}$ value were measured and tension tests on the SMB supports were conducted. A comparison of characteristics after these tests with those from before the tests confirmed that there was no deterioration. These results confirm that the conventional SMB design can support large loads without increasing the amount of superconducting wire. In order to evaluate the stability of the SMB under changing rotational speeds, evaluation equipment for acceleration tests that allow simultaneous levitation/rotation is being developed, which can also evaluate the long term reliability and durability of the HTS coils and cryo-parts of the SMB.

\section{Acknowledgements}

This research was supported to build on results and progress obtained with the Komekurayama prototype FESS, which was subsidized by NEDO as part of the project, "Development of Next Generation Flywheel Power Storage System". The "Development of a Superconducting Magnetic Bearing Able to support Large Loads in a Flywheel Energy Storage System for Railway Applications," was made possible thanks to the generous cooperation of several companies including, Matsuikozai Co., Ltd., Mitsubishi Kogyo Co., Ltd. and Maruwa Electronic Inc. The authors would like to express their deepest gratitude to all parties involved in supporting this work. 


\section{References}

[1] Nagashima, K., Seino, H., Miyazaki, Y., Arai, Y., Sakai, N. and Murakami, M., "Loading force density of a superconducting magnetic bearing using superconducting bulk body and superconducting coils," RTRI Report, Vol.21, No.9, pp.29-34, 2007 (in Japanese).

[2] Hasegawa, H., Matsue, H., Nagashima, K. and Yamashita, T., "Development of superconducting flywheel energy storage systems for demonstrations," RTRI Report, Vol.29, No.11, pp.41-46, 2014 (in Japanese).

[3] Yamashita, T., Ogata, M., Matsue, H., Miyazaki, Y., Sugino, M. and Nagashima, K., "Verification of Reliability of Superconducting Flywheel Energy Storage System and its Application to Railway System," $Q R$ of RTRI, Vol. 58, No. 4, pp 303-310, 2017.

[4] Miyazaki, Y., Mizuno, K., Yamashita. T., Nagashima, K., Nakao, K., Matsuoka, T., "Development of a Superconducting Magnetic Bearing Able to support Large Loads in a Flywheel Energy Storage System for Railway Applications," RTRI Report, Vol. 32, No. 3, pp. 35-40, 2018 (in Japanese).

[5] Shimizu, H., Sawamura, H., Ozawa, K., Miyazaki, K., Mukoyama, S., Nagashima, K., Yamashita, M., "Status of HTS flywheel energy storage system demonstration machine in 2017 Komekurayama," Abstracts of CSSJ Conference, Vol. 95, p. 165, 2017 (in Japanese).

[6] Seino, H., Nagashima, K., Tanaka, Y. and Nakauchi, M., "Study of the magnetic bearing consisting of coupling of superconductors applicable to the support bearing of flywheel energy storage system," RTRI Report, Vol.22, No.11, pp.35-40, 2008 (in Japanese).

[7] Mizuno, K., Sugino, M., Ogata, M., "Experimental Production and Evaluation of Facetrack Coils for Onboard REBCO Magnet," RTRI Report, Vol.29, No.11, pp.11-16, 2015 (in Japanese).

[8] Mizuno, K., Sugino, M., Tanaka, M., Ogata, M., “Development of a Real-scale REBCO Coil for the Demonstration of Magnetomotive force of $700 \mathrm{kA}$," RTRI Report,
Vol.31, No.1, pp.5-10, 2017 (in Japanese).

[9] Mizuno, K., Yamashita, T., Miyazaki, Y., Sakamoto, H., Nakao, K., Matsui, Y., Dohi, T., "Enhance a maximum load of High Temperature Superconducting Magnetic Bearing -New type coil structure can balance low heat with cooling-," Abstracts of CSSJ Conference, Vol.94, p.128, 2017 (in Japanese).

[10]Nakao, K., Sakamoto, H., Mizuno, K., Miyazaki, Y., Yamashita, T., "HTS magnetic bearing for heavy load (1) -New coil design using bonding process-," Abstracts of CSSJ Conference, Vol.95, p.166, 2017 (in Japanese).

[11] Teshima, H., Morita, M., "Recent Progress in Material Technology on RE-Ba-Cu-O Bulk Superconductors," TEION KOGAKU, vol. 45, No. 3, pp 73-80, 2011 (in Japanese).

[12]Morita, M., "Processing and superconducting properties of high-Jc bulk YBaCuO prepared by melt process," Japan Society of Applied Physics, Vol. 62, No. 5, pp. 483-484, 1993.

[13]Mizuno, K., Miyazaki, Y., Yamashita, T., Nakao, K., Sakamoto, H., Shimizu, H., Sawamura, H., Ozawa, T., Matsui, Y., Dohi, T., Uejima, F., "HTS magnetic bearing for heavy load (2) -Results of heavy load tests-," Abstracts of CSSJ Conference, Vol.95, p.167, 2017 (in Japanese).

[14]Yamafuji, K., "Flux Creep in a Superconductor," TEIONKOGAKU, Vol. 25, No. 1, pp. 2-11, 1990 (in Japanese).

[15]Funaki, K., "Basic Approach to High Tc Superconducting Bulks and Their Applications," TEIONKOGAKU, Vol. 28, No. 4, pp. 183-190, 1993.

[16]Murakami, M., "Material Science of High Tc Superconductors," Uchidarokakuho, pp. 171-176, 1999 (in Japanese).

[17]Miyazaki, Y, Yamashita, T., Mizuno, K., Matsui, Y., Dohi, T., Uejima, F., Asano, Y., Kazama, T., Nakao, K., Sakamoto, H., "HTS magnetic bearing for heavy load (3) -Test results of heavy load and reliability for thermal insulating support-," Abstracts of CSSJ Conference, Vol. 95, p.168, 2017 (in Japanese).

\section{Authors}

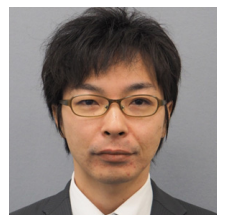

Yoshiki MIYAZAKI, Dr. Eng.

Assistant Senior Researcher, Cryogenic

Systems Laboratory, Maglev Systems

Technology Division,

Research Areas: Cryogenics

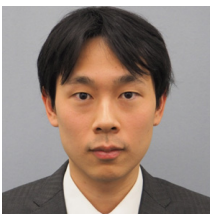

Katsutoshi MIZUNO, Dr. Eng.

Assistant Senior Researcher, Cryogenic

Systems Laboratory, Maglev Systems

Technology Division,

Research Areas: Superconducting Technology

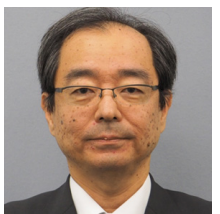

Masafumi Ogata, Dr. Eng.

Senior Chief Researcher, Head of Cryogenic

Systems Laboratory, Maglev Systems

Technology Division,

Research Areas: Superconducting Technology

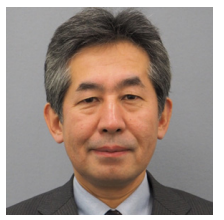

Tomohisa YAMASHITA

Senior Researcher, Maglev Systems

Technology Division,

Research Areas: Superconducting Technology

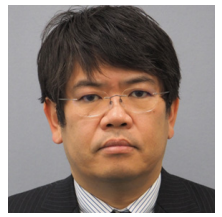

Ken Nagashima, Dr. Eng.

Director, Head of Maglev Systems Technology Division,

Research Areas: Superconducting Technology 